\title{
Effects of decitabine on megakaryocyte maturation in patients with myelodysplastic syndromes
}

\author{
KAI DING* ${ }^{*}$, RONG FU* , HUI LIU* , DEEPAK ANIL NACHNANI and ZONG-HONG SHAO \\ Department of Hematology, General Hospital of Tianjin Medical University, Tianjin 300052, P.R. China
}

Received November 12, 2014; Accepted October 23, 2015

DOI: $10.3892 / 01.2016 .4259$

\begin{abstract}
Thrombocytopenia is a common, often fatal complication experienced by patients with myelodysplastic syndromes (MDS). 5-aza-2'-deoxycytidine (decitabine) has been used to treat MDS patients with thrombocytopenia with a response rate of $45-50 \%$. However, the mechanism of its effects on megakaryocytes remains unclear. In the present study, the effect of decitabine on megakaryocyte maturation was investigated. A total of $20 \mathrm{MDS}$ patients diagnosed with thrombocytopenia were enrolled, including 16 refractory anemia with excess blasts (RAEB)-1 patients and 4 RAEB-2 patients], in addition to 20 leukemia patients that had achieved complete remission and 20 healthy donors. Overall, $65 \%$ of MDS patients exhibited a response to decitabine, with an increase in platelet count identified in $80 \%$ of patients. In the MDS group, the mean platelet count was significantly increased following one cycle of decitabine chemotherapy (36.85 \pm 24.54 vs. $84.90 \pm 61 ; \mathrm{P}=0.001$ ); however, no significant difference in megakaryocyte number was identified prior to and following treatment. Additionally, bone marrow mononuclear cells of the MDS patients were cultured in vitro with various concentrations of decitabine $(0.0,2.0,2.5,3.0 \mu \mathrm{M})$, and cluster of differentiation (CD)41 levels were examined via flow cytometry. The MDS and normal control groups exhibited the highest levels of CD41 expression following treatment with $2.0 \mu \mathrm{M}$ decitabine (mean fluorescence intensity, 294.07士47.34 and $258.95 \pm 28.05$, respectively). In conclusion, these results indicate that the DNA-hypomethylating agent, decitabine, may induce the differentiation and maturation of myelodysplastic megakaryocytes in MDS patients, even at low concentrations. Thus, the repeated administration of decitabine at lower doses in MDS patients may be useful in clinical practice, and may
\end{abstract}

Correspondence to: Professor Rong Fu or Professor Zong-Hong Shao, Department of Hematology, General Hospital of Tianjin Medical University, 154 Anshan Road, Heping, Tianjin 300052, P.R. China E-mail: florai@vip.sina.com

E-mail: shaozonghong@sina.com

*Contributed equally

Key words: myelodysplastic syndromes, decitabine, thrombocytopenia lead to the development of alternative treatments for other diseases of abnormal megakaryocyte differentiation, such as idiopathic thrombocytopenic purpura, however, future studies are required to investigate this.

\section{Introduction}

Myelodysplastic syndromes (MDS) are a heterogeneous group of clonal disorders that originate from early hematopoietic progenitor cells. These syndromes are characterized by maturation defects that lead to ineffective hematopoiesis, resulting in anemia, leucopenia and thrombocytopenia (1). In the USA, the annual incidence rate for MDS is 2-4 cases per 100,000 individuals. Notably, MDS are most common in elderly individuals; the annual MDS incidence rate ranges between 15 and 50 cases per 100,000 individuals in patients aged $>70$ years (1). The majority of patients ultimately succumb to infection and/or hemorrhage. Thrombocytopenia (platelet count, $<100 \times 10^{9} / 1$ ) is common in MDS, occurring in $40-65 \%$ of patients (2). In severe cases of thrombocytopenia and/or hemorrhage, thrombocytopenia in MDS is treated via platelet transfusions. However, platelet transfusions often lead to a brief increase in platelet count and antibodies may be produced subsequent to multiple transfusions, causing human leukocyte antigen-alloimmunization. Furthermore, $\geq 30 \%$ of platelet transfusions result in complications, including bacteremia, graft-versus-host disease, acute pulmonary injury and exacerbated thrombocytopenia $(3,4)$.

5-Aza-2'-deoxycytidine (decitabine) is a known inhibitor of DNA methylation and has been shown to exhibit a marked antileukaemic effect (5). Decitabine has been shown to induce response rates of $45-50 \%$ in elderly high-risk MDS patients, even inducing trilineage response and cytogenetic complete remission (CR) in $\sim 30 \%$ of patients with cytogenetic abnormalities, by increasing platelet count (6). However, the mechanism by which decitabine increases the platelet count in patients with MDS remains unclear (6-9).

The association between decitabine and cellular differentiation was initially identified in the human erythroleukemia cell line, K562. Decitabine was found to induce irreversible hemoglobinization and morphological differentiation in a dose-dependent manner (7,8). A number of previous studies have suggested that the effects of decitabine treatment for thrombocytopenia in MDS patients may be due to enhanced megakaryocyte differentiation (6-12). Therefore, to elucidate 
the mechanisms by which decitabine increases platelet count in patients with MDS, and to investigate the effects of decitabine on megakaryocyte differentiation and platelet release, the present study used cluster of differentiation (CD)41 (integrin, $\alpha \mathrm{IIb}$ ) as a cell surface marker for megakaryocyte maturation (13). Furthermore, the associated clinical data was collected prior to and after decitabine treatment, allowing analysis of the association between the clinical data and laboratory results.

\section{Materials and methods}

Patient characteristics. Patients with a hematological diagnosis of de novo primary MDS were considered eligible for the present study. A total of 20 MDS patients with thrombocytopenia (11 males, 9 females) with a median age of 55 years (range, 35-75 years) were enrolled at the General Hospital of Tianjin Medical University (Tianjin, China) between March 2013 and February 2014. According to the World Health Organization criteria (14), 16 patients exhibited refractory anemia with excess blasts (RAEB)-1 and 4 patients exhibited RAEB-2. Two control groups were also enrolled between March 2013 and February 2014. The first control group included 20 acute myeloid leukemia patients (12 males, 8 females) with a median age of 46 years (range, 20-69 years) who had achieved CR after induction chemotherapy [1 M2, $8 \mathrm{M} 4,6 \mathrm{M} 3$ and 5 M5 patients (15)]. The second control group included 20 healthy donors (10 males, 10 females) with a median age of 40 years (range, 22-57 years). Written informed consent was obtained from all patients and healthy donors, and the study was approved by the Ethics Committee of the General Hospital of Tianjin Medical University (Tianjin, China).

Treatment. All patients were administered with intravenous decitabine (20 mg/m²/day; Xian Janssen Pharmaceutical Ltd., Beijing, China) for 5 consecutive days, according to Kantarjian's protocol proposed by the National Comprehensive Cancer Network (NCCN) (16). After one course of treatment, bone marrow aspiration was performed to assess the effects of decitabine.

Determination of treatment efficacy. MDS patient subtypes, blast cell count and treatment response was determined according to NCCN guidelines (16). After one cycle of decitabine treatment, all the patients underwent bone marrow aspiration to determine treatment efficacy. All bone marrow smears were evaluated by one hematologist. A bone marrow sorting counter (WZR-BM2; Ai Lin, Suzhou, China) was used by the hematologist to count nucleic cells in the bone marrow smears, and a total of 500 nucleic cells were counted in each smear. If the blast cell percentage increased, this was considered as disease progression, and if the blast cell percentage decreased by $<50 \%$, this was considered as a poor response. In all leukemia $\mathrm{CR}$ patients and donors, the blast cell count was $<5 \%$ in the bone marrow.

Megakaryocyte count. The number of megakaryocytes in a $1.5 \times 3.5-\mathrm{cm}$ area of a standard bone marrow smear were determined under microscope (BX53; Olympus Corporation, Tokyo, Japan) using Wright's staining (Sigma-Aldrich,
St. Louis, MO, USA) in all patients and donors, as described previously $(17,18)$. Megakaryocyte morphology was also analyzed in all patients and donors $(17,18)$.

Platelet count. Prior to and following one course of decitabine treatment, patients' peripheral blood platelet count was performed using a CytoFLEX blood cell counter (Beckman Coulter, Inc., Brea, CA, USA).

In vitro induction of primary bone marrow mononuclear cell (BMMNC) differentiation. A total of $5 \mathrm{ml}$ bone marrow aspirate was obtained from each subject at the Department of Hematology (General Hospital of Tianjin Medical University). Overall, 80 bone marrow samples were collected; 40 samples were obtained from MDS patients (prior to and following one cycle of decitabine treatment), 20 samples from the leukemia CR patients and 20 samples from the healthy donors. EDTA (BD Biosciences, San Diego, CA, USA) was added to the aspirate at the time of collection. The samples were washed immediately with sterile phosphate-buffered saline (PBS; BD Biosciences). BMMNCs were separated with Facoil buffer (Sigma-Aldrich) and seeded in a 6-well culture plate. Cells $\left(1.10-1.20 \times 10^{6}\right.$ cells/well) were cultured at $37^{\circ} \mathrm{C}$ in a $1: 1$ mixture of Dulbecco's modified Eagle's medium and Ham's F-12 (Gibco; Thermo Fisher Scientific, Waltham, MA, USA) with $10 \mathrm{ng} / \mathrm{ml}$ recombinant human thrombopoietin (Thermo Fisher Scientific) and $5 \mathrm{ng} / \mathrm{ml}$ stem cell factor (Thermo Fisher Scientific) for 7 days. Cells were counted using a cell count plate (Eppendorf, Hamberg, Germany) prior to and following culture. To assess cell viability Trypan Blue (Sigma-Aldrich) was used to stain cells and only viable cells were counted. Decitabine was added to the wells in a concentration ladder $(0.0,2.0,2.5$ and $3.0 \mu \mathrm{M})$. Megakaryocyte differentiation was evaluated following 7 days of culture at $37^{\circ} \mathrm{C}$ in at atmosphere containing $5 \% \mathrm{CO}_{2}$. At harvesting, $1 \mathrm{ml}$ PBS was added to each well. Following gentle mixing with a plastic pipette $(1 \mathrm{ml}$ pipette; Thermo Fisher Scientific), cells were counted and harvested with the pipette. Megakaryocytes remained suspended in PBS and were incubated with $20 \mu 1$ mouse anti-human CD41 $\alpha$-fluorescein isothiocyanate immunoglobulin $\mathrm{G}$ antibody (ready to use; cat. no. 340929; BD Biosciences) at $4^{\circ} \mathrm{C}$ for $30 \mathrm{~min}$ in the dark. Following incubation, the cells were washed twice with PBS. Approximately 30,000 cells were acquired and analyzed for CD41 expression using fluorescence-activated cell sorting analysis (FACS; FACScalibur ${ }^{\mathrm{TM}}$; BD Biosciences) and CellQuest $^{\mathrm{TM}}$ software version 6.0 (BD Biosciences).

Statistical analysis. All results are expressed as the mean or median \pm standard deviation (SD). Data were analyzed using the independent samples t-test for comparisons between groups. All statistical analyses were performed using SPSS 19.0 software (SPSS Inc., Chicago, IL, USA). $\mathrm{P}<0.05$ were considered to indicate a statistically significant difference.

\section{Results}

In vivo effects of decitabine. In the present study, all MDS patients enrolled were thrombocytopenic at the commencement of therapy. After one cycle of decitabine treatment, an increase in platelet count was identified in 16 patients 

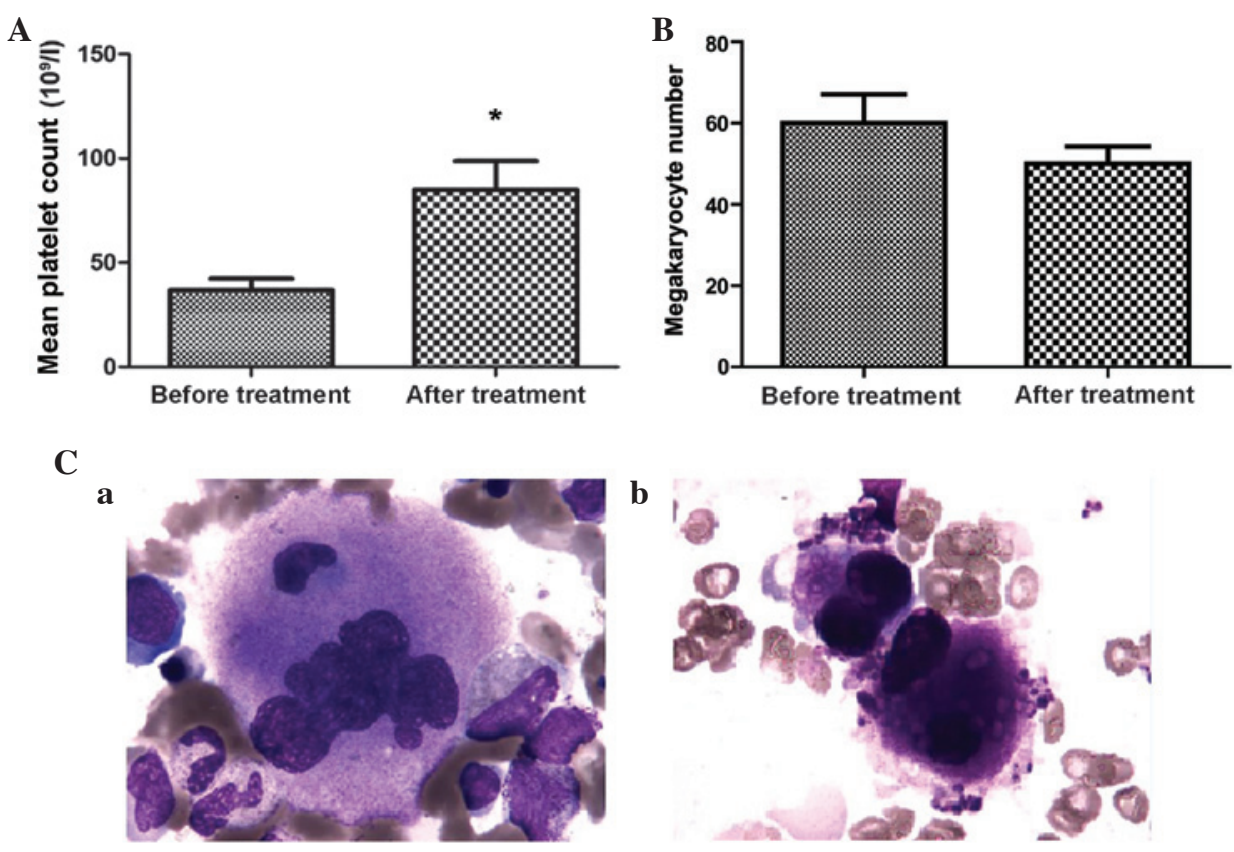

Figure 1. (A) Platelet counts of patients with MDS were significantly elevated after one cycle of 5-aza-2'-deoxycytidine (decitabine) treatment (20 mg/ $\left.\mathrm{m}^{2} / \mathrm{day}\right)$. ${ }^{*} \mathrm{P}=0.001$. Data are expressed as the mean \pm standard deviation. (B) MDS patients' megakaryocyte number prior to and following one cycle of decitabine chemotherapy. Data are expressed as the mean \pm standard deviation. (C) Megakaryocyte count and morphological analysis in a single patient with MDS (magnification, x100; Wright's staining). (Ca) Prior to treatment with decitabine $\left(20 \mathrm{mg} / \mathrm{m}^{2} / \mathrm{day}\right)$, the dysplastic megakaryocyte was immature and the release of platelets was dysfunctional. $(\mathrm{Cb})$ In the same patient's bone marrow after decitabine treatment, a mature megakaryocyte producing platelets was observed. MDS, myelodysplastic syndrome.
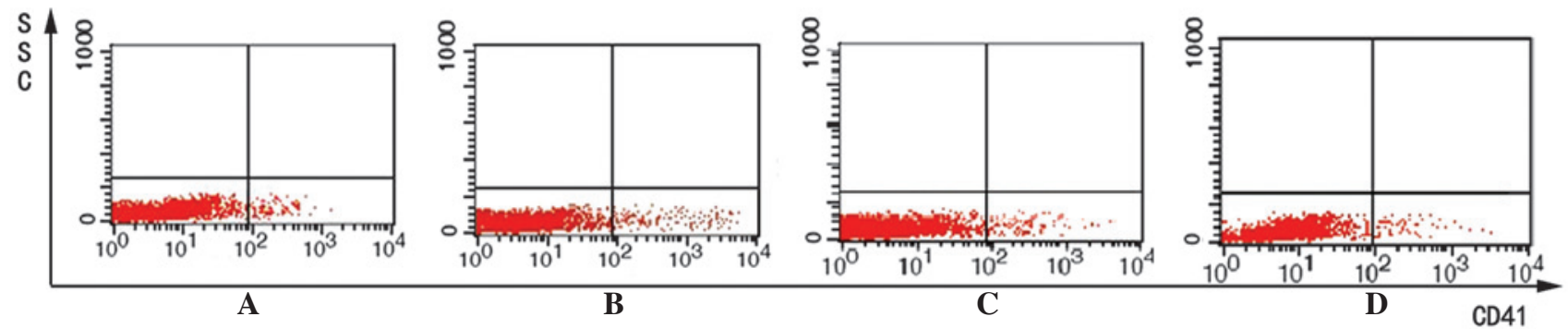

Figure 2. Megakaryocytes in bone marrow samples, detected by flow cytometry. (A) CD41 expression in the bone marrow mononuclear cells of a patient with myelodysplastic syndromes was extremely low prior to 5-aza-2'-deoxycytidine (decitabine) treatment [MFI, 206.73]. (B) CD41 expression increased after treatment with $2.0 \mu \mathrm{M}$ decitabine (MFI, 308.83), and decreased progressively when cultured with (C) $2.5 \mu \mathrm{M}$ (MFI, 288.10) and (D) 3.0 $\mu \mathrm{M}$ (MFI, 256.08) decitabine. CD, cluster of differentiation; MFI, mean fluorescence intensity.

(80\%), however, platelet count remained stable (increased or decreased by $\left.<10 \times 10^{9} / 1\right)$ in 4 patients. The mean platelet counts were significantly increased in the MDS patient group, from $36.85 \pm 24.54 \times 10^{9} / 1$ before treatment to $84.90 \pm 61.85 \times 10^{9} / 1$ after treatment $(\mathrm{P}=0.001$; Fig. $1 \mathrm{~A})$. However, no significant differences in megakaryocyte count were identified in the MDS patient group following one cycle of decitabine therapy $(\mathrm{P}>0.05)$. For the healthy donor group and leukemia $\mathrm{CR}$ patient group, the mean platelet counts were $198.55 \pm 54.25 \times 10^{9} / 1$ and $224.13 \pm 72.33 \times 10^{9} / 1$, respectively, and these patients did not receive any treatment in the present study. Morphological analysis revealed megakaryocyte maturation and the production of platelets following treatment of MDS patients with $20 \mathrm{mg} / \mathrm{m}^{2} /$ day decitabine (Fig. 1B and $\mathrm{C}$ ). Furthermore, in 13 patients, bone marrow blast cell count decreased by $>50 \%$. For $3 / 7$ poorly responded patients, blast cell count increased after one cycle of decitabine chemotherapy. Notably, the platelet count increased to $>30 \times 10^{9} / 1$ in all of the 3 progressed patients (data not shown). In the control group, the size and maturation of maturation of megakaryocytes was normal, and the blast cell percentage was $<0.5 \%$.

In vitro effects of decitabine. To investigate the effect of decitabine on megakaryocyte differentiation, megakaryocytes were cultured in vitro and exposed to various concentrations of decitabine $(0.0,2.0,2.5$ and $3.0 \mu \mathrm{M})$ to identify the optimal concentration required for megakaryocyte maturation. These results may provide a partial guide for the clinical use of decitabine in MDS patients with refractory thrombocytopenia. Briefly, BMMNC cells were incubated with various concentrations of decitabine $(0.0$, 2.0, 2.5 and $3.0 \mu \mathrm{M}$ ) for 7 days. The BMMNC cells were harvested, stained for the megakaryocyte marker CD41, and 
Table I. Mean fluorescence intensity of membrane cluster of differentiation 41 in the bone marrow mononuclear cells of MDS patients, following treatment with decitabine at various concentrations.

\begin{tabular}{llcl}
\hline & \multicolumn{3}{c}{ Mean fluorescence intensity $( \pm \mathrm{SD})$} \\
\cline { 2 - 4 } Decitabine $(\mu \mathrm{M})$ & Control group & Leukemia CR & de novo MDS \\
\hline 0.0 & $284.53 \pm 38.12$ & $318.91 \pm 24.70$ & $226.19 \pm 17.61$ \\
2.0 & $294.07 \pm 47.34$ & $307.42 \pm 55.40$ & $258.95 \pm 28.05$ \\
2.5 & $273.25 \pm 34.26$ & $273.05 \pm 47.54$ & $242.89 \pm 24.11$ \\
3.0 & $272.93 \pm 38.36$ & $232.43 \pm 33.90$ & $224.23 \pm 16.05$
\end{tabular}

MDS, myelodysplastic syndromes; decitabine, 5-aza-2'-deoxycytidine; CR, complete remission; SD, standard deviation.

evaluated by flow cytometry. The mean fluorescence intensity (MFI) of CD41 was compared between the four different treatment groups. In the MDS patients group, the results indicated that $2.0 \mu \mathrm{M}$ decitabine induced the highest expression of CD41 following treatment (MFI, 258.95 $\pm 28.05 ; \mathrm{P}<0.05$; Table I; Fig. 2), however, CD41 expression remained consistently and significantly lower than the healthy control group $(\mathrm{P}<0.05)$. In the leukemia CR patients group, the expression of CD41 was significantly higher than that of the healthy controls prior to decitabine treatment (MFI, 318.91 \pm 24.70 vs. $284.53 \pm 38.12$; $\mathrm{P}<0.05$; Table I); however, CD41 expression significantly decreased in the leukemia CR group with increasing decitabine concentration $(\mathrm{P}<0.05$; Table I). In addition, the cell number of every well was calculated prior to and after cell culture. A total of $1.10-1.20 \times 10^{6}$ cells were seeded in every well. Following culture, no significant differences in cell number were identified in the $0.0,2.0$ or $2.5 \mu \mathrm{M}$ treatment subgroups. However, following treatment with $3.0 \mu \mathrm{M}$ decitabine, the cell numbers of all three groups decreased to $0.5-0.8 \times 10^{6} /$ well and these differences were determined to be significant $(\mathrm{P}<0.05$; Fig. 3 ).

\section{Discussion}

Previously, the treatment of elderly MDS patients with decitabine was found to result in a response rate of $45-50 \%(10,11)$. Increased platelet count is a major response observed in these patients. This is of particular importance, as supportive care involves platelet transfusions, which may be a burden for the patient, often leading to a refractory response to expensive platelet transfusions (1-3).

In the current study, after one cycle of decitabine treatment, a platelet response was observed in 16/20 (80\%) MDS patients, with an mean increase of $>30 \times 10^{9} / 1$. In 2004 , Van den Bosch et al (12) investigated 162 high-risk MDS patients. The authors reported that $58 \%$ of thrombocytopenic patients exhibited a platelet response following one cycle of decitabine therapy, and $69 \%$ of patients with a low platelet count exhibited a response during therapy. In addition, an increase in platelet count was found to be preceded by a positive trilineage response. The platelet responses observed in MDS patients following decitabine treatment in the present study and the study by Van den Bosch et al (12) (80 and $65 \%$, respectively) were higher than the previously reported

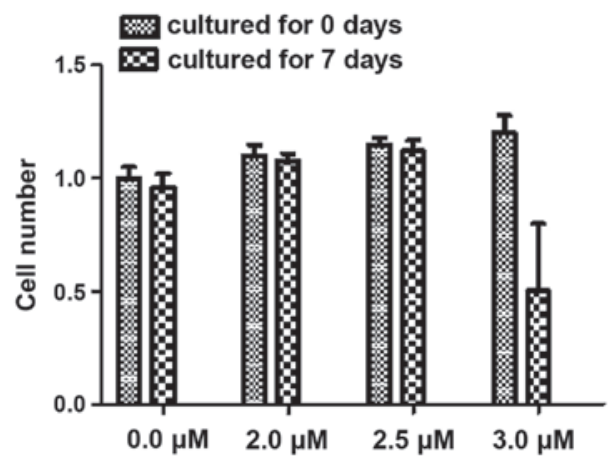

Figure 3. Cultured cell number (presented as the mean \pm standard deviation) in different concentration groups $(2.0,2.5$ and $3.0 \mu \mathrm{M})$ prior to and following 7 days culture.

platelet response rates of $45-50 \%(6,10,11)$. Therefore, it is hypothesized that the megakaryocytic lineage pathways affected by decitabine may be independent from its effects on other lineages. Notably, in the current study, after one cycle of decitabine, the three progressed patients exhibited an evident platelet response, which enforces our hypothesis. Alternatively, dysplastic megakaryocytes may exhibit more sensitivity and thus respond to treatment earlier than other cell lineages (12). The maturation of megakaryocytes involves endomitosis (19), whereby the latter stages of mitosis are bypassed to allow an increase in DNA content and size of cells; therefore, megakaryocytes may be more sensitive to hypomethylation agents, such as decitabine, due to high levels of DNA replication (9).

In the present study, a concentration ladder of decitabine was used for in vitro cell culture. The results indicated that the MFI of CD41 was highest in the $2.0 \mu \mathrm{M}$ decitabine subgroup (Fig. 2; Table I). Wang et al (9) performed a similar experiment in vitro using the mouse cell line, L8057, and demonstrated that decitabine induced the highest expression of CD41 at a concentration of $2.5 \mu \mathrm{M}$. Notably, these concentrations of decitabine are lower than the concentration used clinically, according to NCCN guidelines (16). CD41 is the surface marker of the megakaryocytic lineage which represents megakaryocyte maturation (20). In the current study, the higher concentration subgroup $(3.0 \mu \mathrm{M})$ expressed a lower MFI and a decreased cell number after 7 days culture, which may be a result of severe cytotoxic side 
effects of decitabine. However, the $3.0 \mu \mathrm{M}$ subgroup, which approximates to the clinical dose used, resulted in higher CD41 expression than the $0.0 \mu \mathrm{M}$ decitabine treatment group. These results indicate that a therapeutic regimen using a lower concentration of decitabine may be of clinical use for the induction chemotherapy of MDS patients. Furthermore, in the present study, although CD41 expression was significantly increased in the MDS group following treatment with $2.0 \mu \mathrm{M}$ decitabine, the expression levels remained lower than those observed in the two control groups, which indicates that additional decitabine consolidation chemotherapy may be required for MDS patients following treatment with induction chemotherapy.

In the present study, the megakaryocyte number was calculated for the MDS patient group prior to and after one cycle of decitabine chemotherapy, however, no significant differences were identified. These results indicate that the increases in platelet counts and CD41 expression observed were due to the improvement of the quality of megakaryocytes, rather than megakaryocyte number. Thus, we hypothesize that decitabine affects the megakaryocytic lineage via the induction of differentiation and maturation of the lineage. Furthermore, maturation of megakaryocytes was observed directly via morphological comparison of patients' bone marrow smears prior to and after decitabine chemotherapy (Fig. 1B).

MDS is a malignant cloning disease. Typically, a small number of normal and dysplastic clones usually coexist in the bone marrow of MDS patients, $(21,22)$. In the current study, megakaryocyte differentiation in healthy donors was also induced by decitabine, as indicated by increased expression of CD41, which peaked in the $2.0 \mu \mathrm{M}$ subgroup (Table I). Similarly, Momparler et al (23) observed a 2-3-fold increase in the platelet count of patients treated for metastatic lung cancer using the same chemotherapeutic agent. In addition, in sickle cell anemia patients treated with even lower decitabine doses (0.15-0.30 mg/kg), an increase in hemoglobin F levels and concomitant increases in platelet counts were observed (24). These results indicate that the normal clones that exist in the bone marrow of MDS patients may also be induced by decitabine, which may explain the platelet response observed following decitabine treatment.

In conclusion, decitabine, as a DNA-hypomethylating agent, appears to induce the differentiation and maturation of myelodysplastic megakaryocytes in MDS patients, even at low concentrations. Therefore, the repeated administration of decitabine at lower doses in MDS patients may be useful in clinical practice, and may lead to the development of alternative treatments for other diseases of abnormal megakaryocyte differentiation, such as idiopathic thrombocytopenic purpura, however, future studies are required to investigate this.

\section{Acknowledgements}

This study was partially supported by The National Natural Science Foundation of China (grant nos. 81170472, 81370607 and 30971285), the Natural Science Foundation of Tianjin (grant nos. 14JCYBJC25400, 14JCYBJC27200), the Tianjin Cancer Major Projects Research Plan (grant nos. 12ZCDZSY17900 and 12ZCDZSY18000), the National Public Health Grand
Research Foundation (grant no. 201202017) and the Tianjin Medical University Fund (grant no. 2011KYQ15).

\section{References}

1. Steensma DP and Tefferi A: The myelodysplastic syndrome(s): A perspective and review highlighting current controversies. Leuk Res 27: 95-120, 2003.

2. Kantarjian H, Giles F, List A, Lyons R, Sekeres MA, Pierce S, Deuson $\mathrm{R}$ and Leveque J: The incidence and impact of thrombocytopenia in myelodysplastic syndromes. Cancer 109: 1705-1714, 2007.

3. Webb JJ and Anderson KC: Risks, costs and alternatives to platelet transfusion. Leuk Lymphoma 34: 71-84, 1999.

4. List A, Kurtin S, Roe DJ, Buresh A, Mahadevan D, Fuchs D, Rimsza L, Heaton R, Knight R and Zeldis JB: Efficacy of lenalidomide in myelodysplastic syndromes. N Engl J Med 352: 549-557, 2005.

5. Richel DJ, Colly LP, Kluin-Nelemans JC and Willemze R: The antileukemic activity of 5-aza-2-deoxycytidine (Aza-dC) in patients with relapsed and resistant leukaemia. Br J Cancer 64: 144-148, 1991.

6. Lübbert M, Wijermans P, Kunzmann R, Verhoef G, Bosly A, Ravoet C, Andre $\mathrm{M}$ and Ferrant A: Cytogenetic responses in high-risk myelodysplastic syndromes following low-dose treatment with the DNA methylation inhibitor 5-aza-2-deoxycytidine. Br J Haematol 114: 349-357, 2001.

7. de Vos D and van Overveld W: Decitabine: A historical review of the development of an epigenetic drug. Ann Hematol 84 (Suppl 1): S3-S8, 2005.

8. Hennessy BT, Garcia-Manero G, Kantarjian HM and Giles FJ: DNA methylation in haematological malignancies: The role of decitabine. Expert Opin Investig Drugs 12: 1985-1993, 2003.

9. Wang J, Yi Z, Wang S and Li Z: The effect of decitabine on megakaryocyte maturation and platelet release. Thromb Haemost 106: 337-343, 2011.

10. Wijermans P, Krulder JW, Huygens PC and Neve P: Continous infusion of low dose 5-aza-2'-deoxycytidine in elderly patients with high-risk myelodysplastic syndromes. Leukemia 11: 1-5, 1997.

11. Wijermans P, Lübbert M, Verhoef G, Bosly A, Ravoet C, Andre M and Ferrant A: Low dose 5-aza-2'-deoxycytidine, a DNA-hypomethylating agent for the treatment of high-risk myelodysplastic syndromes: A multicenter phase II study in elderly patients. J Clin Oncol 18: 956-962, 2000.

12. Van den Bosch J, Lübbert M, Verhoef G and Wijermans PW: The effects of 5-aza-2'-deoxycytidine (Decitabine) on the platelet count in patients with intermediate and high-risk myelodysplastic syndromes. Leuk Res 28:785-790, 2004.

13. Peng HY and Liao HF: Staurosporine induces megakaryocytic differentiation through the upregulation of JAK/Stat3 signaling pathway. Ann Hematol 90: 1017-1029, 2011.

14. Brunning R, Orazi A, Germing U, et al: Myelodysplastic syndromes. In: World Health Organization Classification of Tumours of Haematopoietic and Lymphoid Tissue. Swerdlow S, Campo E, Harris NL, et al. (eds.). 4th edition. IARC Press, Lyon, France, pp88-103, 2008.

15. Cheson BD, Bennett JM, Kopecky KJ, Büchner T, Wiliman CL, Estey EH, Schiffer CA, Doehner H, Tallman MS, Lister TA, et al; International Working Group for Diagnosis, Standardization of Response Criteria, Treatment Outcomes, and Reporting Standards for Therapeutic Trials in Acute Myeloid Leukemia: Revised recommendations of the International working group for diagnosis, standardization of response criteria, treatment outcomes, and reporting standards for therapeutic trials in acute myeloid leukemia. J Clin Oncol 21: 4642-4649, 2003.

16. Greenberg PL, Attar E, Bennett JM, et al; National Comprehensive Cancer Network: NCCN Clinical Practice Guidelines in Oncology: Myelodysplastic syndromes. J Natl Compr Canc Netw 9: 30-56, 2011.

17. Gajendra S, Jha B, Goel S, Sahni T, Sharma R, Shariq M, Jaiswal S and Sachdev R: Leishman and Giemsa stain: A new reliable staining technique for blood/bone marrow smears. Int J Lab Hematol: Jul 30, 2015 (Epub ahead of print).

18. Mori H, Niikura H, Terada $\mathrm{H}$ and Fujita K: Morphological analysis of the megakaryocytes in myelodysplastic syndrome. Rinsho Byori 38: 1347-1352, 1990 (In Japanese). 
19. Bluteau D, Lordier L, Di Stefano A, Chang Y, Raslova H, Debili N and Vainchenker W: Regulation of megakaryocyte maturation and platelet formation. J Thromb Haemost 7 (Suppl 1): 227-234, 2009.

20. Gao X, Wu J, Zou W and Dai Y: Two ellagic acids isolated from roots of sanguisorba officinalis L. Promote hematopoietic progenitor cell proliferation and megakaryocyte differentiation. Molecules 19: 5448-5458, 2014.

21. Giagounidis A, Mufti GJ, Fenaux P, Germing U, List A and MacBeth KJ: Lenalidomide as a disease-modifying agent in patients with del (5q) myelodysplastic syndromes: Linking mechanism of action to clinical outcomes. Ann Hematol 93:1-11, 2014.
22. Valent P, Bain BJ, Bennett JM, Wimazal F, Sperr WR, Mufti G and Horny HP: Idiopathic cytopenia of undetermined significance (ICUS) and idiopathic dysplasia of uncertain significance (IDUS) and their distinction from low risk MDS. Leuk Res 36: $1-5,2012$.

23. Momparler RL, Bouffard DY, Momparler LF, Dionne J, Belangerc K and Ayoub J. Pilot phase I-II study on 5-aza-2-deoxycytidine (Decitabine) in patients with metastatic lung cancer. Anticancer Drugs 8: 358-368, 1997.

24. Koshy M, Dorn L, Bressler L, Molokie R, Lavelle D, Talischy N, Hoffman R, van Overveld W and DeSimone J: 5-Aza-2-deoxycytidine and foetal haemoglobin induction in sickle cell anaemia. Blood 96: 2379-2384, 2000. 